\title{
Dialysis Free Protocol for Some End Stage Renal Disease Patients (Khosroshahi Protocol)
}

\author{
Hamid Tayebi Khosroshahi ${ }^{1}$ and Kamyar Kalantar-zadeh ${ }^{2}$ \\ ${ }^{1}$ Department of Internal Medicine, Dialysis Center, Imam Reza Hospital, Tabriz University of \\ Medical Sciences, Tabriz, Iran; ${ }^{2}$ Harold Simmons Center for Kidney Disease Research and \\ Epidemiology at Harbor-UCLA Medical Center, Torrance, CA, USA
}

Corresponding Author: Hamid Tayebi Khosroshahi, MD, Professor, Chronic Kidney Disease Research Center, Tabriz University of Medical Sciences, Tabriz, Iran

Submission date: May 15, 2012, Acceptance date: August 28, 2012; Publication date: August 30,2012

\section{ABSTRACT:}

Background: The number of patients with End Stage Renal Disease (ESRD) is growing annually around the world. Provision of renal replacement therapy in the form of dialysis and transplant is relatively expensive. Recent studies have shown no survival benefit of early initiation of dialysis. Given recent outcome data of the timing of dialysis treatment and the expenses and logistics of hemodialysis procedure have stimulated research on alternative strategies. The aim of this study is to introduce another type of renal replacement therapy for selective patients with advanced chronic kidney disease (CKD).

Methods: In a case series we used a so-called "dialysis free protocol" consisting of a low protein diet, uremic toxin adsorbents, and prebiotics for selective ESRD patients who had dialysis access constraints or did not agree placement of hemodialysis vascular access or peritoneal dialysis catheter.

Findings: clinical and biochemical results of this study showed that these patients were not deteriorating during the study period and Blood Urea Nitrogen (Bun) \& serum creatinin levels were not elevated in these months. Participating patients were relatively well throughout the study without signs of florid uremia and without a need for emergent or urgent dialysis.

Conclusion: We conclude that our proposed dialysis free protocol reduces the need for dialysis treatment at least transiently. Whether this protocol can reduce the need for dialysis treatment requires additional studies.

Key words: End Stage Renal Disease, Low Protein Diet, Prebiotics, Activated Charcoal

INTRODUCTION: The number of patients who require renal replacement therapy is increasing all around the globe. The rising need for dialysis and renal transplant can only be 
dealt with at enormous expense. It is clear that resources to support this expansive growth are limited, both in developing and developed countries (1). The few alternatively available treatments for pre-dialysis patients and the expensive hemodialysis procedure has stimulated research on alternative strategies which might help delay the need for dialysis and improve overall health $(2,3)$. The potential benefit of low protein diets for uremic patients has long been debated. Diet modification in uremia should relieve uremic symptoms, correct some of the complications seen in kidney failure, and preserve or improve the nutritional state of patients (4-6). Prebiotics on the other hand, as a non-digestible food beneficially affects the host by selectively stimulating the growth and /or activity of one or a limited number of bacteria (usually bifidobacteria and lactobacilli) in the colon and thus improves host health (7-9). Previous experience suggests that oral administration of a probiotic formulation of selected microbial strains may extend renoprotection via intraintestinal extraction of toxic waste solutes in patients with chronic kidney disease (CKD) stages 3 and 4 (10).

There have been several reports in addition that suggest the administration of activated charcoal and AST-120 retards the progression of CRF in both uremic rats and undialysed uremic patients. At present, AST-120 is widely used as an approved drug in Japan for the treatment of approximately 20,000 undialysed uremic patients to delay the progression of CRF. However, AST-120 is not yet available outside Japan (11-12). The aim of our proposed dialysis free protocol is to introduce another type of renal replacement therapy for selective patients with advanced CKD.

METHODS: In a case series we used a so-called "dialysis free protocol" including low protein diet, uremic toxin adsorbents and prebiotics for selective ESRD patients who have dialysis access constraints or did not agree to the placement of a hemodialysis vascular access port or a peritoneal dialysis catheter. Our protocol includes a three directional way to reduce or eliminate the nitrogenous waste product in ESRD patients, and delay or alleviate the need for dialysis for at least a short period in these patients [1- low protein diet, 0.6-0.8 g/kg with high biologic value, supplemented with carbohydrates and polyunsaturated fat. 2- Activated charcoal, at a dose of 15 grams (powder), was diluted in a small glass of water as an absorbent of nitrogenous waste product (intestinal dialysis). It was administered after each meal, far from other medications. 3- Lactulose as an accessible prebiotic, $30 \mathrm{ml}(20 \mathrm{~g}) 3$ time per day in order to change the gastrointestinal microflora to health bacteria and reduction of nitrogenous waste product by good bacteria for absorption to the blood]. BUN, Creatinin, $\mathrm{Na}+\mathrm{K}+$, lipids $\mathrm{CBC}$, were measured periodically during the study. Patient's clinical status was controlled for any deterioration or need to dialyze. All data were collected at the end of study for analysis.

\section{CASES:}

Case 1: A 25 year old man with ESRD secondary to Alport syndrome started hemodialysis two sessions ( 8 hours) per week 3 months ago. His sister received a renal transplant 3 years ago. He received a renal transplant after 6 months dialysis from a living unrelated donor, but the allograft was removed due to technical problems and allograft vein thrombosis without function after 5 days. Due to dialysis incompliance and access failure, he fit our dialysis free protocol. Renal function was tested (blood urea, serum creatinin), electrolytes were evaluated every two weeks and CBC, serum lipids and albumin were tested monthly. After the six month dialysis free period, he was well and active and in clinically and para-clinically 
reasonable condition. Physical examination revealed an active thin man with $\mathrm{Bp}=140 / 85$ mmhg without uremic symptoms and signs. He was on other medications for treatment of anemia (erythropoietin, folate, vitamins), renal osteodystrophy (active vitamin D, calcium carbonate,) and hypertension. His body mass index (BMI) was 18.3 that not differ during the study. Now he is a candidate for re-transplantation (Table 1).

Table 1. Renal replacement therapy for selective patients with advanced chronic kidney disease

\begin{tabular}{|c|c|c|c|c|c|}
\hline & Case 1 & Case 2 & Case 3 & Case 4 & Case 5 \\
\hline \multicolumn{6}{|c|}{ Blood Urea mg/dl } \\
\hline Baseline & 149 & 140 & 322 & 220 & 186 \\
\hline Month 2 & 155 & 169 & 414 & 205 & 208 \\
\hline Month 4 & 140 & 145 & 350 & 305 & 192 \\
\hline Month 6 & 150 & 150 & 202 & 201 & 168 \\
\hline Month 8 & & 115 & & & \\
\hline Month 10 & & 130 & & & \\
\hline \multicolumn{6}{|c|}{$\begin{array}{l}\text { Serum Creatinine } \\
\mathrm{mg} / \mathrm{dl}\end{array}$} \\
\hline Baseline & 6.8 & 6.1 & 22 & 8.1 & 7.6 \\
\hline Month 2 & 6.5 & 5.8 & 17.2 & 8.5 & 5.3 \\
\hline Month 4 & 6.0 & 6.6 & 13.9 & 7.4 & 6.0 \\
\hline Month 6 & 5.6 & 6.7 & 12.9 & 8.5 & 4.8 \\
\hline Month 8 & & 6 & & & \\
\hline Month 10 & & 6 & & & \\
\hline \multicolumn{6}{|c|}{ Hemoglobin g/dl } \\
\hline Baseline & 8.9 & 9.8 & 8.8 & 11.0 & 8.2 \\
\hline Month 2 & 10.8 & 10.9 & 9.2 & 11.8 & 9.4 \\
\hline Month 4 & 11.0 & 11.2 & 9.9 & 13.0 & 9.7 \\
\hline Month 6 & 11.6 & 12.0 & 10.3 & 11.8 & 10.8 \\
\hline Month 8 & & 10.6 & & & \\
\hline Month 10 & & 10.7 & & & \\
\hline \multicolumn{6}{|c|}{ Cholesterol mg/dl } \\
\hline Baseline & 169 & 150 & 170 & 140 & 112 \\
\hline Month 2 & 158 & 138 & 174 & 127 & 121 \\
\hline Month 4 & 170 & 153 & 165 & 129 & 108 \\
\hline Month 6 & 153 & 130 & 167 & 146 & 127 \\
\hline Month 8 & & 137 & & & \\
\hline Month 10 & & 158 & & & \\
\hline
\end{tabular}

Case 2: A 60 year old woman with ESRD due to hypertension started chronic hemodialysis 15 months ago. She was on dialysis for 5 months with access problems. After that, due to access failures and disagreement with continuing of dialysis, she fit our dialysis free protocol. She was clinically under control, and renal function tests, electrolytes, CBC, and nutritional status (lipids and albumin) were measured biweekly and monthly according to clinical status. She was well without uremic symptoms after 10 months on this regimen. She was also on 
other medications for treatment of anemia, renal osteodystrophy edema as in Case 1. Her BMI was stable during dialysis freedom period and persistently more than 25 (Table 1).

Case 3: A 29 year old man with ESRD due to Alport syndrome started hemodialysis due to uremia and need for renal replacement therapy. He was on hemodialysis for 3 months. Due to Atrio-enous fistula failure, hemodialysis was stopped. He did not agree with short term central venous catheter insertion. Due to access failure and with patient's agreement, dialysis freedom protocol was started. He was clinically under control and renal function tests, electrolytes, $\mathrm{CBC}$ and nutritional status were evaluated biweekly and monthly as above patients. Other replacement therapy included the treatment of anemia, renal osteodistrophy, and hypertension, and vitamins were administered as the patients on continues dialysis. $\mathrm{He}$ was at good status after 3 month dialysis freedom protocol. His BMI was also not differ during the study and was 18 . He started hemodialysis via Atrio-Venous fistula due to non compliance to continue dialysis free protocol (Table 1).

Case 4: A 24 year old girl with ESRD with unknown cause admitted to our university hospital due to complications of dialysis. Jugular vein catheter was inserted and hemodialysis started and continued for 5 sessions. Due to catheter malfunction, hemodialysis was discontinued and her dialysis catheter was removed. Due to patient incompliance with hemodialysis catheter re-insertion, dialysis free protocol was begun. Routine laboratory tests including renal function tests were performed monthly and the patient was clinically evaluated for uremic symptoms, nutritional status and need for dialysis. She was also on other medications including replacement therapy for anemia (erythropoietin, folate, vitamins) renal osteodistrophy (calcitriol, calcium carbonate) and hypertension. This protocol was continued for 6 months, and the patient was well and without need to dialyze. Her BMI was also 26 and he had $4 \mathrm{~kg}$ weight gains during the study. Now she is candidate for renal transplantation from living unrelated donor (Table 1).

Case 5: A 54 year old woman with ESRD due to unknown cause was referred to our university clinic for to begin dialysis. She had no dialysis access and disagreed with transient intravenous catheter insertion. She had nausea and her estimated GFR was about 6\%. Due to incompliance with dialysis initiation, and acceptance of our protocol, dialysis free protocol was started. Renal function tests, $\mathrm{Hb}$, and nutritional status were evaluated periodically. She was also on other medications for treatment of anemia, hypertension, edema, and renal osteodistrophy. After 6 months she was in a good clinical and laboratory condition without uremia symptoms and need for emergency dialysis. Her BMI was more than 25 without weight loss during the study period (Table 1).

DISCUSSION: In many geographic areas most patients with ESRD are treated with chronic dialysis or kidney transplantation. However, in many others areas social, ethical, and financial considerations, in addition to inadequate facilities, government funding, and public acceptance affects renal replacement therapy. Nevertheless, the lack of effective RRT in many areas of the world should not mean that patients with ESRD must be left without suitable care alternatives (13). There are many methods being developed by many investigators from differing cultures and geographic areas for patients who can not continue routine RRT (especially dialysis) for many reasons. We used a regimen of low protein diet, 
with high biologic value in our protocol. The use of low protein diet, high biologic value proteins, and special formula, is one of the methods that could potentially alleviate the need for RRT in predialysis state (4-6). Low protein diet with the addition of Acacia gum (1 $\mathrm{g} / \mathrm{kg} /$ day) is another regimen that has recently been reported to be a successful alternative to dialysis for some ESRD patients $(13,14)$. One other part of our protocol is the use of adsorbents with a reasonable affinity to intestinal nitrogenous waste product. Activated charcoal in doses of 30 to 45 gram daily (15 g in a glass of water with each meal) was administered. Intestinal dialysis and use of adsorbent such as activated charcoal or AST 120 with some affinity to intestinal nitrogenous waste products, is another known method of decreasing the need for dialysis in ESRD patients (15). Studies of intestinal dialysis have shown a daily flow of 71 grams of urea, 2.9 grams of creatinine, 2.5 grams of uric acid and 2.0 grams of phosphate into the intestinal fluid (16). Accordingly, various invasive and noninvasive attempts including external gut fistula, intestinal dialysis, induced diarrhea, and administration of oral sorbents and/or encapsulated urease enzyme have been made to extract uremic waste from the gastrointestinal tract (2,3,7-15).

The second part of our protocol was the use of prebiotic. We used lactulose as a prebiotic in this study. In one study, supplemental nutrition with probiotic activity that improved gastrointestinal flora, and diets rich in prebiotics (such as lactulose, inulin, and oligofroctose) have been used for delaying the need for dialysis in some areas. Various therapies have been developed to regulate the complex bacterial fermentation processes (17) Younes and coworkers demonstrated that fermentable carbohydrates alter colonic bacterial fermentation in CKD (18-19). In other studies, they demonstrated that the prebiotic oligofructose-enriched inulin reduced urinary $p$-cresol excretion (including its sulfate conjugate) in healthy volunteers. Whether fermentable carbohydrates, such as oligofructose and inulin, affect serum concentrations of protein-bound uraemic retention solutes in patients with CKD is not known (20). To our knowledge, this is the first report regarding the use of this combination of regimens (low protein diet, adsorbents, and prebiotics) in order to reduce the need for dialysis in ESRD patients. Our criteria for selecting the patients for protocol were adult ESRD patients without indication of emergent need for dialysis, and agreed to and tolerated the use of this regimen. This protocol is safe and useful for at least: patients with ESRD who have access failure and who are candidates for renal transplantation during the next few months, patients who need transient intravenous catheter insertion for a few months to facilitate Atrio-Venous fistula maturation, andpatients who disagree with or socioeconomically unable to begin dialysis. Our protocol also saves more money. The cost of one monthof dialysis in our country is at least $\$ 1,000$, and central venous catheter insertion is an invasive and expensive procedure with several complications. Dialysis is also an invasive procedure with some morbidity and mortality. Our protocol cost is less than $10 \%$ of dialysis cost, and is noninvasive.

CONCLUSION: It seems that dialysis freedom protocol is safe and even necessary for some ESRD patients who have access problems, are economically disadvantaged, or who disagree with beginning dialysis and/or are candidates for renal transplantation within the next few months.

Competing interests: The authors declare that they have no competing interests. 
Acknowledgements: We acknowledge the contribution of patient's families' and our dialysis world personnel to this study.

\section{REFERENCES:}

1. Paul E. de Jong, Marije van der Velde, Ron T. Gansevoort, and Carmine Zoccali Screening for Chronic Kidney Disease: Where Does Europe Go? Clin J Am Soc Nephrol 2008, 3: 616-623.

2. Natarajan Ranganathan, Beena Patel, Pari Ranganathan, Joseph Marczely, Rahul Dheer, Tushar Chordia, Stephen R. Dunn, and Eli A. Friedman. Probiotic Amelioration of Azotemia in 5/6th Nephrectomized Sprague-Dawley Rats Research Article TheScientificWorldJOURNAL . 2005, 5, 652-66.

3. Bj”orn K. I. Meijers, Vicky De Preter, Kristin Verbeke, Yves Vanrenterghem1 and Pieter Evenepoel. $p$-Cresyl sulfate serum concentrations in haemodialysis patients are reduced by the prebiotic oligofructose-enriched inulin Nephrol Dial Transplant 2009 1of 6 doi: 10.1093/ndt/gfq580

4. Sintra Eyre, RD, and Per-Ola Attman, MD, PHD. Protein restriction and body composition in renal disease. Journal of Renal Nutrition, 2008, Vol 18,No 2(March), pp 167- 186

5. David J, Hirsch, MD, FRCPC. Limited Protein Diet: a means of delaying the progression of chronic renal disease.CAN MED ASSOC J, 1985. Vol 132, April 15, pp 913- 917.

6. AM EL Nahas,A Masters-Thomas, S A Brady, K Farrington, V Wilkinson. Selective effect of low protein diet in chronic renal disease. BRITISH MEDICAL JOURNAL, 1984,17 NOVEMBER. PP 1337- 1341

7. Ranganathan, Natarajan; Patel, Beena G.; Ranganathan, Pari; Marczely, Joseph; Dheer, Rahul; Pechenyak, Bohdan; Dunn, Stephen R.; Verstraete, Willy; Decroos, Karel; Mehta, Raj; Friedman, Eli A. In Vitro and In Vivo Assessment of Intraintestinal Bacteriotherapy in Chronic Kidney Disease. ASAIO Journal: January/February 2006 - Volume 52 - Issue 1 - pp 70-79

8. Marcel Roberfroid, Prebiotics: The Concept Revised, The Journal of Nutrition (supplement). 2007, pp 830S- 837S

9. John H Cumming, Georg T Macfarlene, and Hans N Englyst. Prebiotic Digestion and Fermentation. Am J Clin Nutr 2001; 73( suppl): 415S- 20S

10. Natarajan Ranganathan · Pari Ranganathan · Eli A. Friedman · Anthony Joseph · Barbara Delano - David S. Goldfarb et al Pilot Study of Probiotic Dietary Supplementation for Promoting Healthy Kidney Function in Patients with Chronic Kidney Disease. Adv Ther (2010) 27(9):634-647

11. Takashi Miyazaki, Isao Aoyama, Michihito Ise, Hisao Seo and Toshimitsu Niwa. An oral sorbent reduces overload of indoxyl sulphate and gene expression of TGF- $\beta 1$ in uraemic rat kidneys.(Nephrol Dial Transplant, 2000, 15:1773-1781

12. Musso CG, Michelangelo H, Reynaldi J, Martinez B, Vidal F, Quevedo M, Parot M, Waisman G, Algranati L, INTESTINAL DIALYSIS IN VERY OLD PATIENTS. Rev Electron Biomed / Electron J Biomed 2008;1:77-78

13. Aamir Jalal Al Mosawi, Six-year dialysis freedom in end-stage renal disease Clin Exp Nephrol_Japanese Society of Nephrology 2009. Volume 13, Number 5, 494-500

14. Aamir Jalal Al-Mosawi. Acacia gum supplementation of a low-protein diet in children 
with end-stage renal disease. Pediatr Nephrol (2004) 19:1156-1159

15. Eli A. Friedman. Can the bowel substitute for the kidney in advanced renal failure? Current Medical Research and Opinion. 2009,VOL. 25, NO. 8, 1913-1918

16. Sparks, R. E. D. Shigella and Salmonella were tested for with Violet Red Bile Glucose (VRBG) agar fluid. Kidney Int. Suppl. 1975 Suppl 3, 7:373-376

17. Guarner F, Malagelada JR. Gut flora in health and disease. Lancet. 2003; 361: 512519

18. Younes H, Remesy C, Behr S et al. Fermentable carbohydrate exerts a urea-lowering effect in normal and nephrectomized rats. Am J Physiol 1997; 272: G515-G521

19. Younes $\mathrm{H}$, Egret $\mathrm{N}$, Hadj-Abdelkader $\mathrm{M}$ et al. Fermentable carbohydrate supplementation alters nitrogen excretion in chronic renal failure. J Ren Nutr 2006; 16: $67-74$

20. De Preter V, Vanhoutte T, Huys G et al. Effects of Lactobacillus casei Shirota, Bifidobacterium breve, and oligofructose-enriched inulin on colonic nitrogen-protein metabolism in healthy humans. Am J Physiol Gastrointest Liver Physiol 2007; 292: G358-G368 